Clarke, P. H. (1955). J. gen. Microbiol. 12, 337-343

\title{
Methods for Determining the Biochemical Activities of Micro-organisms as applied to Classification
}

\author{
By PATRICIA H. CLARKE \\ Department of Biochemistry, University College, London
}

We all know what we mean by biochemical methods, although it is unlikely that we all mean the same. We may differ in the biochemical criteria we use for classification, and also in the techniques we prefer for employing them.

The main methods in general use can be divided as in Table 1. In addition to providing information about the synthetic abilities of micro-organisms, nutritional studies enable us to prepare chemically-defined media. Most of

\section{Table 1. Biochemical methods used in classification}

$\begin{array}{ll}1 & \text { Minimal growth requirements } \\ 2 & \begin{array}{c}\text { Substrate utilization for growth } \\ \text { and energy source }\end{array} \\ 3 & \text { End products of metabolism } \\ 4 & \begin{array}{c}\text { Identification of characteristic } \\ \text { compounds including enzymes }\end{array}\end{array}$

\author{
Nutritional studies \\ Including metabolic pathways to \\ some extent \\ From complex media; single \\ carbohydrates or any other \\ compound \\ Gums, capsules, toxins, storage \\ products, etc.
}

the tests are concerned with metabolic breakdown (groups 2 and 3 ), and here the varied and often extreme metabolic behaviour shown by micro-organisms makes it possible to describe them by the substrates they metabolize and the compounds they produce. Many micro-organisms have characteristic and biologically unusual end products of metabolism: for example, Thiobacillus thio-oxidans which produces up to $\mathrm{N} / 10$ sulphuric acid; so that we often encounter organisms, the biochemical equivalent in curious development of the sabre-toothed tigers.

Using simple and established methods for each of these groups, we could very rapidly find out this information about the habits of Staphylococcus aureus. For growth and energy it requires organic carbon and $\mathrm{CO}_{2}$. It will not grow on an ammonium salt medium, and requires the addition of a source of growth factors to a protein hydrolysate. Further study will show that it requires a certain number of amino acids and growth factors. We find that it ferments a number of carbohydrates with the production of acid but no gas; it produces acetoin from glucose, and ammonia but not indole from peptone; reduces nitrates to nitrites; liquefies gelatin; and produces a coagulase, haemolysins and an enterotoxin.

From the point of view of classification in the abstract, it is irrelevant how difficult it is to determine any property about a living organism if it can be 
done at all, and if it can be repeated. With micro-organisms, it is a distinct advantage to be able to study large numbers of strains, to be sure that the character is not confined to a few only. This does not mean that observations should be limited to growing cultures in test tubes. Techniques may involve microtests, plate cultures, washed suspensions or extracts, but the ideal to be aimed at is an elegant simplicity of technique.

These are the methods I am concerned with here-methods in constant use by microbiologists whereby some of the biochemical characteristics of a large number of strains can be studied, and I shall make a few tentative suggestions for possible developments. Most of my examples will be drawn from the heterotrophic group of bacteria, because they have been more intensively studied than any other group of micro-organisms.

Many of the tests we use have been borrowed from the diagnostic laboratory. The main requirements for diagnosis are a limited number of speedy and reliable tests which will pick out the suspected organisms. In applying biochemical tests to classification we also want to find out as much as we can about the biochemical behaviour of the organisms which would have escaped through the diagnostic sieve and to assess the significance of our findings. Not all our tests are of equal importance. In devising a rational scheme of classification we should like to assign a hierarchy of subdivisions according to the relative importance of the different characters, but this is not easy to do.

Table 2. Breakdown of glucose and other carbon compounds

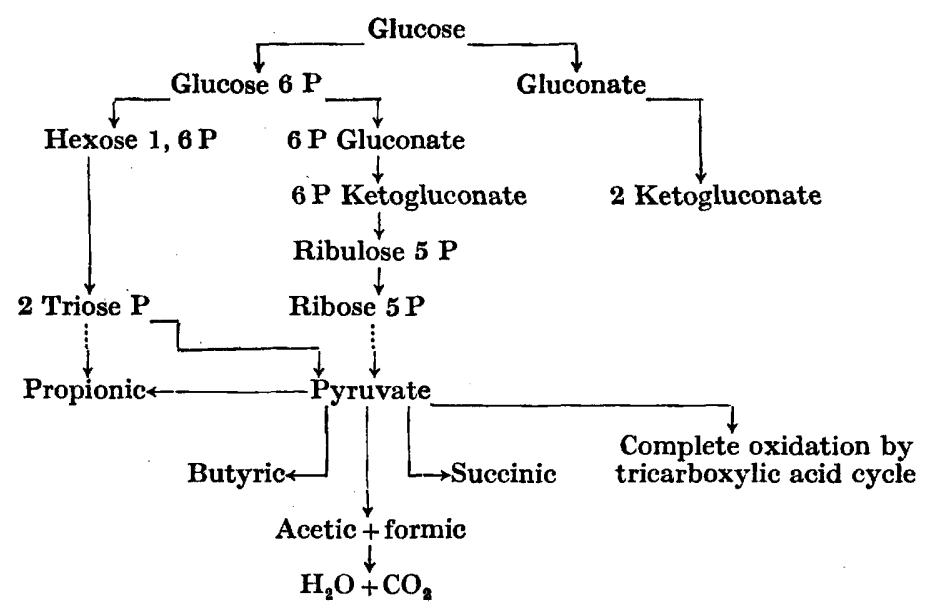

In the breakdown of glucose and other carbon compounds (Table 2) many fungi and a few bacteria such as the pseudomonads may metabolize glucose by direct oxidation, other fungi and bacteria use the oxidative monophosphate pathway, and the lactobacilli an anaerobic variation of it. Algae and some bacteria use the glycolytic pathway, with or without a final stage of oxidation by the tricarboxylic acid cycle or otherwise. These divisions by metabolic pathways are more fundamental than divisions on the basis of whether two disaccharides 
such as maltose and sucrose are both metabolized by two organisms. More than one pathway may be present in one organism; and if so, various factors will determine how much glucose is metabolized in each way.

Have we any tests which will show these differences? Barron \& Friedmann (1941) showed that glucose utilization by Pseudomonas aeruginosa was not inhibited by fluoride, suggesting that it was not metabolized exclusively by the glycolytic pathway. This could be applied as a test to detect organisms not dependent entirely on the glycolytic route of glucose breakdown. Hugh \& Leifson (1953) suggest a simple method for distinguishing bacteria which primarily ferment glucose from those which metabolize it by oxidation. They incubate two tubes of medium so that one will show anaerobic metabolism and the other aerobic metabolism. From their results they divide the organisms they have studied into three groups (Table 3): (1) organisms which neither

Table 3. Use of glucose metabolism in classification (after Hugh \& Leifson)

\begin{tabular}{lccc}
\multicolumn{1}{c}{ Species } & Aerobic & Anaerobic & \\
Alkaligenes faecalis & - & - & (1) Non-oxidizer, non-fermenter \\
$\begin{array}{l}\text { Pseudomonas aeruginosa } \\
\text { Bacterium anitratum }\end{array}$ & A & - & (2) Oxidizers, non-fermenters \\
Shigella dysenteriae & A & - & \\
Vibrio cholerae & A & A & $(3 a)$ Fermenters (anaerogenic) \\
Salmonella enteritidis & A & A & \\
Escherichia coli & AG & AG & $(3 b)$ Fermenters (aerogenic) \\
Klebsiella aerogenes & AG & AG &
\end{tabular}

oxidize nor ferment glucose; (2) organisms which oxidize but do not ferment; (3) anaerogenic and aerogenic fermenting organisms. Again, the ability to attack gluconate has been shown to be a useful classificatory character. Haynes (1951), in studying the characteristically oxidative pseudomonads, tested a large number of strains for their ability to produce 2-ketogluconate from potassium gluconate. De Ley (1953) has shown that several bacterial species metabolize gluconate; and Shaw (personal communication) found it a useful character in subdividing the Enterobacteriaceae, although the route and end products were not known.

The end products of metabolism of carbohydrate are usually recorded as acid, or acid and gas. For some purposes, and in considering closely related organisms, acid and gas may be a sufficient statement; but it is often important to know 'what acid and what gas'. The propionibacteria, the anaerobic butyric bacteria, and many other groups of bacteria and fungi, have wellknown and characteristic end products of fermentation. In general, apart from a few special cases such as the production of acetoin from glucose, there are no simple tests for end products, although for more detailed study new methods are available such as the elegant gas liquid partition chromatography method of James \& Martin (1952) for fatty acids.

A simple and general test for the ability to use the simple organic acids is 
based on the development of an alkaline reaction when the organisms are grown on a simple medium containing the sodium salts of the organic acids. Kauffmann (1951) used this method for testing the activity of the Enterobacteriaceae towards citrate, tartrate, etc.; and Leifson (1933) used malonate in this way to differentiate Escherichia coli from Klebsiella aerogenes. It is a method of very wide application.

It is unlikely that these simple tests, used alone, will reveal fundamental biochemical differences; they are no substitute for the intensive biochemical study of enzyme systems. It does not follow, for example, that if an organism does not grow on a simple citrate medium, it is completely unable to metabolize citrate. Escherichia coli is said to be citrate-negative, it produces no visible growth on a citrate medium; yet most strains will grow if supplied with a little glutamic acid as well, and cell-free extracts from washed suspensions have been shown to metabolize citrate and to contain the citric acid cycle enzymes. The difference between citrate-negative and citrate-positive organisms may be one of permeability, but it is none the less useful in classification.

Compared with carbohydrates, the activity of micro-organisms towards nitrogen compounds has been neglected for classification. The usual tests include only the digestion of gelatin and, as far as amino acids are concerned, the production of indole from tryptophan and $\mathrm{H}_{2} \mathrm{~S}$ from sulphur-containing amino acids in complex organic media. The last has been confused by the habit of some authors of adding inorganic sulphur compounds such as sulphite or thiosulphate to their media.

But micro-organisms can attack nitrogen compounds in almost every conceivable way, and we might look here for some new tests. For example, we could exploit the differences in amino acid metabolism. Gale (1946) found that the amino acid decarboxylases of the species he examined had a definite and limited distribution. Sharpe (1949) used a test based on tyrosine decarboxylase to separate Streptococcus lactis and S. faecalis. Møller (1954) has recently surveyed several hundred strains of species of the Enterobacteriaceae, and found it possible to distinguish groups by their decarboxylases. King (1953) found that Proteus vulgaris produced decarboxylases for valine and leucine; this fitted with Proom \& Woiwod's (1951) amine test for Proteus species.

\section{Table 4. Use of amino acid decarboxylases in classification}

Bacterial amino acid decarboxylases

$\begin{array}{llllllllll}\text { E. coli } & + & + & + & + & + & + & + & - & - \\ \text { K. aerogenes } & + & - & + & + & - & - & - & - & - \\ \text { P. vulgaris } & - & - & - & + & - & + & - & + & + \\ \text { S. faecalis } & - & - & - & - & + & - & - & - & - \\ \text { C. welchii } & + & - & - & - & - & + & + & - & -\end{array}$

Hist. = histidine, Arg. = arginine, Lys.= lysine, Orn. = ornithine, Tyr. =tyrosine, Glut. A. = glutamic acid, Asp. = aspartic acid, Leuc. = leucine, Val. = valine. 
Stumpf \& Green (1944) described an L-amino acid oxidase of Proteus vulgaris which could attack most of the natural amino acids by oxidative deamination. Clarke \& Shaw (1954) have tested for this enzyme among the Enterobacteriaceae with phenylalanine as the substrate, using both growing cultures and a very simple test with washed suspension. Among several hundred strains of various species shown in Table 5 they found that this enzyme and also leucine decarboxylase were found at an appreciable level only among Proteus species and the Providence group. The L-amino acid oxidase of Proteus is a case of excessive production of an enzyme by a group of bacteria. It can be detected in some other species at a very much lower level. These two sets of tests are more precise than the older description of Proteus as 'producing amines', and 'producing ammonia from peptone'.

Table 5. Division of Enterobacteriaceae by the phenyl alanine test

\begin{tabular}{lrr} 
& $\begin{array}{c}\text { Oxidative deamination } \\
\text { of phenyl alanine }\end{array}$ \\
\multicolumn{1}{c}{ Organism } & + & - \\
Proteus spp. & 62 & 0 \\
Providence group & 125 & 0 \\
Arizona group & 0 & 54 \\
Bethesda-Ballerup group & 0 & 9 \\
E. coli I & 0 & $\mathbf{4 3}$ \\
Klebsiella spp. & 0 & $\mathbf{6 8}$ \\
Shigella spp. & 0 & $\mathbf{2 0}$ \\
Salmonella spp. & 0 & $\mathbf{2 1 1}$
\end{tabular}

I mention these studies because the methods are very simple tests for single enzymes, and can readily be fitted into a series. A few tests for single enzymes are already widely used, such as urea splitting by urease, or indole production from tryptophan, although the latter is usually carried out on complex mediapeptone or broth. The advantages of using a defined substrate and/or chemically defined media are obvious. There is considerable scope for developing many more methods both for classification and also for diagnostic tests on these lines.

Most of the methods to which I have referred are empirical. I am suggesting that having applied to classification our knowledge of energy sources, synthetic abilities and metabolic pathways, we subsequently utilize any character which varies in a consistent way, even if its metabolic significance is not known. So that on the one hand we want to develop tests which are related to the more fundamental differences, and on the other to exploit any enzyme system of uneven distribution for classification at the finer subdivisions.

The possession of a common enzyme is not by itself evidence for close relationship of organisms. It may only mean that the organisms live in the same kind of environment and metabolize the same substrates. Studies of enzyme distribution must be evaluated against the other characters of the organism including morphology, serology and any toxin production. It would 
be artificial to rank these characters in a rigid order of importance for all groups. It can be argued that fundamentally they are all biochemical. The toxins whose modes of action have been elucidated have been shown to be enzymes and there is no reason to suppose that collagenase and hyaluronidase are any less or more important than urease or glutamic acid decarboxylase. Even the demonstration of morphological features depends on the presence of the necessary chemical grouping to react with the appropriate stains.

What sort of results can we expect to get? If we carry out a series of quantitative studies on any particular enzyme system on a number of strains of the same species, we will find a very large variation in activity. Even if we take single cells, we will find that without any artificial aids to mutation they may throw off variants with more, less or none of the activity we are investigating. If we start with an organism possessing a certain number of biochemical characters by means of which inter alia we define it, then it may lose a certain number of these characters, and we will still describe it as a variant of the original species. Where we draw the line is a matter of taste, and the more organisms we study in detail, the more blurred become the lines between species and between genera. The biochemical plasticity of micro-organisms, enabling them to multiply in more than one environment, results in this accumulation of surplus enzyme potential, some of which is readily lost. We may therefore find that we can best express some of the biochemical characters on a statistical basis, or more simply describe characters as invariably positive, invariably negative, usually so, or variable.

Finally, although it is a difficult subject for general discussion of this kind, I must refer briefly to experimental details. Many differences of opinion about biochemical reactions, for example whether or not Tetrahymena ferments certain sugars, have been due to differences, often slight, in the methods used. Over a limited range of organisms it is often possible to devise a standard method, and when this can be done it is more important to have an agreed method than an optimum one. In general, one can say that no description of biochemical characters is complete unless the method is indicated. The Bergey Manual (1948), for example, gives no indication of the validity of the characters they list and some of them mean very little. 'Nitrates reduced' or 'nitrates not reduced' is a familiar expression, but as Kluyver (1953) pointed out, the absence of a positive reaction for nitrite after an organism has been grown for a few days on a broth or agar containing potassium nitrate has more than one interpretation. It may mean that no nitrate has been reduced to nitrite or that reduction has proceeded beyond the nitrite stage. In this case we can easily arrange to get more precise information about what is happening to the nitrate, and the more unequivocal we are able to make these biochemical tests the more use they will be for classification. 


\section{REFERENCES}

Barron, E. S. G. \& Friedemann, T. E. (1941). Studies on biological oxidations. XIV. Oxidation by micro-organisms which do not ferment glucose. J. biol. Chem. 137, 593.

Bergey's Manual of Determinative Bacteriology (1948). 6th ed. Edited by Breed, R. S., Murray, E. G. D. \& Hitchens, A. P. London: Baillière, Tindal and Cox.

Clarke, P. H. \& Shaw, C. (1954). Biochemical tests for Proteus and Providence cultures. J. gen. Microbiol. 10, i.

DE LEY, J. (1953). The direct oxidation of carbohydrates and related compounds by micro organisms. VI Congr. int. Microbiol. 1, 126.

Gale, E. F. (1946). The bacterial amino acid decarboxylases. Advanc. Enzymol. 6, 1.

Haynes, W. C. (1951). Pseudomonas aeruginosa-its characterization and identification. J. gen. Microbiol. 5, 939.

Hugh, R. \& LEIFson, E. (1953). The taxonomic significance of fermentative versus oxidative metabolism of carbohydrates by various Gram-negative bacteria. J. Bact. 66, 24.

JAMES, A. T. \& MARTIN, A. J. P. (1952). Gas-liquid partition chromatography: the separation and microestimation of volatile fatty acids from formic acid to dodecanoic acid. Biochem. J. 50, 679.

Kauffmann, F. (1951). Enterobacteriaceae. Copenhagen: Eijnar Munksgaard.

KrNG, H. K. (1953). The decarboxylation of valine and leucine by washed suspensions of Proteus vulgaris. Biochem. J. 54, xi.

KLUYver, A. J. (1953). Some aspects of nitrate reduction. VI Congr. int. Microbiol. Symp. Microbial Metabolism, p. 71.

Leifson, E. (1933). The fermentation of sodium malonate as a means of differentiating Aerobacter and Escherichia. J. Bact. 26, 329.

Møluer, V. (1954). Activity determination of amino acid decarboxylases in Enterobacteriaceae. Acta path. microbiol. scand. 34, 102, 115.

Proom, H. \& Worwod, A. J. (1951). Amine production in the genus Proteus. J. gen. Microbiol. 5, 930.

Sharpe, M. E. (1949). Some biochemical characteristics of Group D streptococci isolated from infants' faeces, with special reference to their tyrosine decarboxylase activity. Proc. Soc. appl. Bact. 11, 13.

StumpF, P. K. \& Green, E. D. (1944). L-Amino acid oxidase of Proteus vulgaris. J. biol. Chem. 153, 387. 